\title{
TRACE LEVEL DETERMINATION AND QUANTIFICATION OF POTENTIAL GENOTOXIC IMPURITIES IN DASATINIB DRUG SUBSTANCE BY UHPLC/INFINITY LC
}

\author{
N. BALAJI ${ }^{1 *}$, SAYEEDA SULTANA ${ }^{2}$ \\ ${ }^{1,2}$ Department of Chemistry, St. Peter's University, Avadi, Chennai 600054, Tamil Nadu, India \\ Email: priyabalan8380@gmail.com
}

Received: 11 Jul 2016 Revised and Accepted: 12 Aug 2016

\section{ABSTRACT}

Objective: A simple, cost-effective and mass compatible ultra-high fast performance liquid chromatographic (Agilent-Infinity LC 1290) method has been developed and validated for the determination of potentially genotoxic impurities in dasatinib active pharmaceutical ingredients.

Methods: This method comprises the determination of three possible genotoxic impurities in dasatinib. The mobile phase is trifluoroacetic acid, acetonitrile and water with linear gradient elution curve number 6 . The column used for the development and validation is zorbax RRHD eclipse plus C18 with the length of $50 \mathrm{~mm}$, the internal diameter of $2.1 \mathrm{~mm}$ and particle size of 1.8 microns.

Results: The limit of detection of the potential genotoxic impurities are less than $0.1 \mu \mathrm{g} / \mathrm{ml}$ with respect to dasatinib test concentration of $1000 \mu \mathrm{g} / \mathrm{ml}$. The limit of quantification of the potential genotoxic impurities is less than $0.3 \mu \mathrm{g} / \mathrm{ml}$ with respect to dasatinib test concentration of $1000 \mu \mathrm{g} / \mathrm{ml}$.

Conclusion: This method has been validated as per ICH guidelines Q2 (R1). These three potential mutagenic impurities are not degradant impurities of dasatinib and its only process related impurities. The method development has been approached using the QbD principle.

Keywords: Genotoxic impurities, Dasatinib, UPLC, UHPLC, QbD, Infinity-LC, Validation

(C) 2016 The Authors. Published by Innovare Academic Sciences Pvt Ltd. This is an open access article under the CC BY license (http://creativecommons. org/licenses/by/4. 0/) DOI: http://dx.doi.org/10.22159/ijpps.2016v8i10.14020

\section{INTRODUCTION}

Sprycel (dasatinib) is indicated for the treatment of adults with newly diagnosed Philadelphia chromosome-positive $(\mathrm{Ph}+)$ chronic myeloid leukemia (CML) in chronic phase. The effectiveness is based on cytogenetic response and major molecular response rates. The trial is ongoing, and further data will be required to determine longterm outcome. Chronic, accelerated, or myeloid or lymphoid blast phase $\mathrm{Ph}+\mathrm{CML}$ with resistance or intolerance to prior therapy including imatinib. Philadelphia chromosome-positive acute lymphoblastic leukemia (Ph+ALL) with resistance or intolerance to prior therapy. The recommended starting dosage of sprycel for chronic phase CML is $100 \mathrm{mg}$ administered orally once daily.

The recommended starting dosage of Sprycel for accelerated phase CML, myeloid or lymphoid blast phase CML, or Ph+ALL is $140 \mathrm{mg}$ administered orally once daily. Tablets should not be crushed or cut; they should be swallowed whole. Sprycel can be taken with or without a meal, either in the morning or in the evening. In clinical studies, treatment with Sprycel was continued until disease progression or until no longer tolerated by the patient.

The effect of stopping treatment after the achievement of a complete cytogenetic response (CCyR) has not been investigated. It is also being evaluated for use in numerous other cancers, including advanced prostate cancer. The dasatinib is chemically described as $\mathrm{N}$-(2-chloro6-methylphenyl)-2-[[6-[4-(2-hydroxyethyl)-l-piperazinyl]-2-methyl-4pyrimidinyl] amino]-5-thiazole carboxamide. It is approved in USFDA as SPRYCEL and is chemically mentioned in the label as N-(2-chloro-6methylphenyl)-2-[[6-[4-(2-hydroxyethyl)-1-piperazinyl]-2-methyl-4pyrimidinyl] amino]-5-thiazolecarboxamide, monohydrate.

As per the literature of this product [3-17, 22-24], no one has reported the UHPLC method for the determination of potentially genotoxic impurities in dasatinib, and this is the novelty of the article.

\section{Synthetic process}

The dasatinib has been synthesized in three steps. The first step comprises the reaction of 2-tert-butoxycarbonylamino-thiazole-5- carboxylic acid (compound A) with that of 2-chloro-6-methylphenylamine in the presence of $\mathrm{N}, \mathrm{N}$-dimethylformamide, oxalyl chloride, dichloromethane, di-isopropylethylamine and trifluoroacetic acid forms N-(2-chloro-6-methyl-phenyl)-3-ethoxyacrylamide (imp-2). The second step involves the reaction with the imp-2, in the presence of $\mathrm{N}$-bromosuccinimide, 1, 4-dioxane, water and thiourea forms 2-amino-thiazole-5-carboxylic acid-(2-chloro-6methyl-phenyl)-amide (imp-3). The third step includes; the formation of dasatinib from imp-3 reacts with 4, 6-dichloro-2methyl-pyrimidine, sodium-t-butoxide, tetrahydrofuran followed by the addition of 2-piperazine-1-yl-ethanol, 1, 4-dioxane and diisopropylethylamine. The synthetic process for the preparation of dasatinib has been shown in fig. 1.

\section{Genotoxicity and carcinogenicity study}

From the evaluation study of genotoxic and carcinogenic impurities as per the above can be removed and replaced with Fig. 1 synthetic scheme of the dasatinib; imp-1 has been given an alert for potential carcinogen based on QSAR [19-20]. The imp-2 and imp-3 have been considered as a genotoxic carcinogen as per structural alert. These alerts have been taken from the software Toxtree (Estimation of toxic hazard-A decision tree approach) version 2.6.6. The maximum daily dosage of dasatinib is about $140 \mathrm{mg}$. The threshold of toxicological concern (TTC) limit could be $10 \mathrm{ppm}$ as per the calculation provided in ICH guideline M7 and based acceptable intake of $1.5 \mu \mathrm{g} /$ day was considered to be protective for a lifetime of daily exposure [25]. Remaining other compounds was not genotoxic or carcinogen because of the negative in structural alert/QSAR [1920]. The structures of potential genotoxic impurities were shown in fig. 2.

\section{MATERIALS AND METHODS}

\section{Materials}

Dasatinib standard has gifted by Techno chemicals limited, India. The imp-1 was supplied by TCI chemicals India private limited. The imp-2 and imp-3 were synthesized in St. Peter's university chemical laboratory. Trifluoroacetic acid and acetonitrile bought from fisher scientific. HPLC grade water was used, prepared from elga water 
purification system, metrohm. The 1290 Infinity binpump (G4220A) as a pump, 1290 Infinity ALS (G4226A) as an autosampler, 1290 Infinity TCC (G1316C) as column thermostat and 1290 Infinity DAD (G4212A) as a detector. The output signal was monitored and processed by agilent open lab CDS software on an intel core i3 computer (Dell). Water baths equipped with MV controller (amkette analytics, ANM alliance) were used for hydrolytic studies. Stability studies were carried out in a humidity chamber (MACK pharma tech, mumbai, India) and photolytic studies were carried out in a photostability chamber (Thermolab photostability chamber, India). Thermal stability studies were performed in a dry air oven (Amkette analytics, ANM alliance).

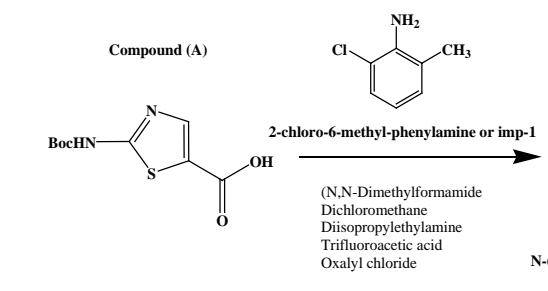

2-tert-butoxycarbonylamino-thiazole-5-carboxylic acid

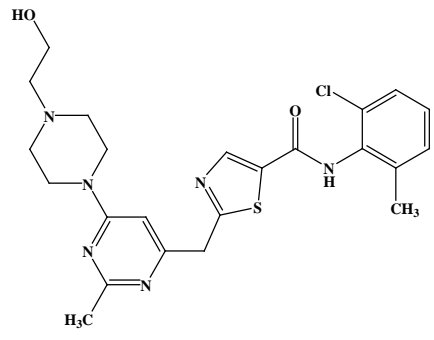

Dasatinib
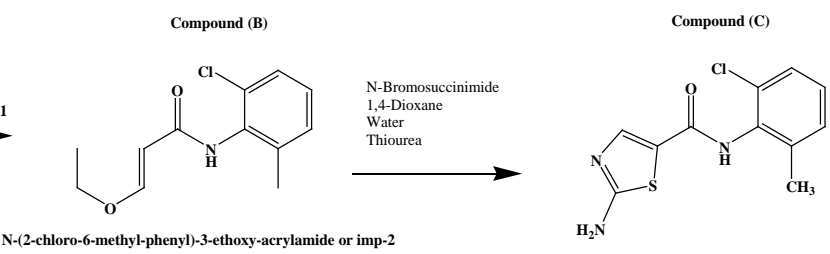

Fig. 1: Synthetic process for the preparation of dasatinib<smiles>Cc1cccc(Cl)c1N</smiles>

2-chloro-6-methyl-phenylamine or imp-1<smiles>CCO/C=C/C(=O)Nc1c(C)cccc1Cl</smiles>

N-(2-chloro-6-methyl-phenyl)-3-ethoxy-acrylamide or imp-2<smiles>Cc1cccc(Cl)c1NC(=O)c1cnc(N)s1</smiles>

2-amino-thiazole-5-carboxylic acid (2-chloro-6-methyl-phenyl)-amide or imp-3

Fig. 2: The structures of potentially genotoxic impurities in synthetic dasatinib process

\section{Methods}

Chromatographic conditions for development screening (phase-1)

The UHPLC columns used for development were zorbax RRHD SB Aq, zorbax RRHD bonus-RP, zorbax RRHD eclipse plus C8, zorbax
RRHD eclipse plus phenyl-hexyl, zorbax PLRP-S and zorbax RRHD eclipse plus C18. The column dimensions were $50 \mathrm{~mm}$ of length, 2.1 $\mathrm{mm}$ internal diameter with $1.8 \mu \mathrm{m}$ particles. Acetonitrile was used as mobile phase-A, and methanol was used as mobile phase-B. Dilute 1 $\mathrm{ml}$ of trifluoroacetic acid in $1000 \mathrm{ml}$ of HPLC grade water used as mobile phase-D1 ( $\mathrm{pH} \mathrm{1.5)} \mathrm{and} \mathrm{dilute} 1 \mathrm{ml}$ of ammonium hydroxide in $1000 \mathrm{ml}$ of HPLC grade water used as mobile phase-D2 (pH 10.5). The total flow rate was $0.6 \mathrm{ml} \mathrm{min}^{-1}$. The column temperature was $30^{\circ} \mathrm{C}$. The gradient time was $5 \mathrm{~min}$ and the wavelength of detection was monitored at a wavelength of $258 \mathrm{~nm}$. The injection volume was $2 \mu$ l. The variables were stationary phase, gradient endpoint \% organic, mobile phase $\mathrm{pH} 1.5$ to 10.5 and other parameters were kept constant.

\section{Chromatographic conditions for development optimization (phase-2)}

The UHPLC column used for development was zorbax RRHD eclipse plus $\mathrm{C} 18$. The column dimensions were $50 \mathrm{~mm}$ of length, $2.1 \mathrm{~mm}$ internal diameter with $1.8 \mu \mathrm{m}$ particles. Acetonitrile was used as mobile phase-A; dilute $1 \mathrm{ml}$ of trifluoroacetic acid in $1000 \mathrm{ml}$ of HPLC grade water used as mobile phase-D1 ( $\mathrm{pH}$ 1.5). The gradient point was $87.5 \%$ acetonitrile.

\section{Chromatographic conditions for validation}

The UHPLC column used for development and validation was a Zorbax RRHD eclipse plus C18, $50 \mathrm{~mm}$ of length, $2.1 \mathrm{~mm}$ internal diameter with $1.8 \mu \mathrm{m}$ particles. Dilute $1 \mathrm{ml}$ of trifluoroacetic acid in $1000 \mathrm{ml}$ of HPLC grade water used as mobile phase-A and mobile phase-B as $100 \%$ acetonitrile. The total flow rate was $0.6 \mathrm{ml} / \mathrm{min}$. The gradient program is given as time $/ \% \mathrm{~B}$ of mobile phase- $\mathrm{B}$ viz., $0.00 / 10.0 \%, 0.20 / 10.0 \%, 2.00 / 30.0 \%, 4.00 / 40.0 \%, 5.00 / 75.0 \%$, $6.50 / 75.0 \%, 7.00 / 10.0 \%$ and $8.00 / 10.0 \%$. The column temperature was sustained at $30{ }^{\circ} \mathrm{C}$ and the wavelength of detection was 
monitored at a wavelength of $258 \mathrm{~nm}$. The injection volume was $2 \mu \mathrm{l}$. Dilute $2 \mathrm{ml}$ of trifluoroacetic acid in $1000 \mathrm{ml}$ of HPLC grade methanol used as sample diluent. The standard solution was injected six times for $\%$ RSD purpose. The sample solution was injected twice for the trace level quantification purpose. The compound-A, B and dasatinib have been included with that of imp-1, imp- 2 and imp-3 in the standard preparation to know the column efficiency and specificity of the method.

\section{Preparation of solutions}

The standard solution was prepared $10 \mathrm{mg}$ of imp-1, imp-2, imp-3, and compound-A, B and dasatinib in $100 \mathrm{ml}$ sample diluent and dissolved using ultrasonication. Further, this solution was diluted 10 $\mathrm{ml}$ to $100 \mathrm{ml}$ with sample diluent $(10 \mu \mathrm{g} / \mathrm{ml}$ with respect to the test concentration of $1000 \mu \mathrm{g} / \mathrm{ml})$. The sample solution was prepared $10000 \mathrm{mg}$ of dasatinib in $10 \mathrm{ml}$ sample diluent and ultra-sonicated for $5 \mathrm{~min}$ and filtered, centrifuged at- $20^{\circ} \mathrm{C}$ for $15 \mathrm{~min}$ and the clear upper part of the solution was injected.

\section{Analytical method development}

\section{Phase 1: screening}

Method development was performed using an Agilent infinity LC II system, open lab CDS method development software. The equipment was equipped with a 6-position column manager and a select solvent valve to enable full method development capability in one system. The initial screening varied column chemistries having SB-Aq, bonus-RP, C8, phenyl-hexyl, PLRP-S and C18 base particles for maximum selectivity. Organic modifier (acetonitrile or methanol) was screened varying the gradient endpoint from $50 \%$ to $100 \%$ organic; over a mobile phase $\mathrm{pH}$ range from 1.5 to 10.5 .

Using these parameters; an experimental design was generated, including randomization and replicate injections. The design generated encompassed the entire knowledge space defined by the constants and variables entered during the experimental setup. A partial factorial statistical design was selected by the software to obtain the maximum amount of information with the least number of experimental runs. The experimental design was transmitted to open lab CDS software where all methods, method sets, and sample sets were automatically generated and ready to run.

After initial integration and processing results from the screening analysis for dasatinib were imported back into open lab CDS software and processed to generate an initial method for subsequent optimization. For the standard solution, a trifluoroacetic acid in water/acetonitrile gradient at $\mathrm{pH} 1.5$ with an $87.5 \%$ acetonitrile gradient endpoint on an eclipse plus C18 column was found to be optimal. The method developed was compatible with mass spectrometric detection and was directly transferred to UHPLCMS to identify and for the confirmation of potentially genotoxic impurities rapidly.

\section{Phase 2: Method optimization}

The initial method was further optimized in a second experiment where secondary effectors such as column temperature, injection volume, flow rate and gradient slope (modified using gradient time) were varied. A new experimental design was generated by open lab CDS software, new methods and sample sets were automatically created.

After processing data; the final optimized method was generated, demonstrating the method that best meets the success criteria defined. In the case of the standard solution separation, an improvement in the tailing of peaks was seen along with better resolution of baseline impurity peaks. Data can also be visualized in 3D plots given fig. 3.

\section{Mobile phase-A selection}

Dasatinib drug substance has three ionization constants 6.8, 3.1 and 10.8 [26] and experimental $\log$ P value of 1.8 [27] and water solubility of $0.0128 \mathrm{mg} / \mathrm{ml}$. The aim of the chromatographic method was to achieve the separation of imp-1, imp-2, imp-3, compound-A, $B$ and the main component dasatinib. Various experiments were performed with different buffers such as phosphate buffer, ammonium acetate and ammonium formate. The $\log \mathrm{P}$ of the compound is 1.8. At higher $\mathrm{pH}$ of the mobile phase, early elution of the components are observed. Whereas at lower $\mathrm{pH}$, baseline drift and disturbance and peak symmetry is good. Hence, trifluoroacetic acid used as mobile phase-A.
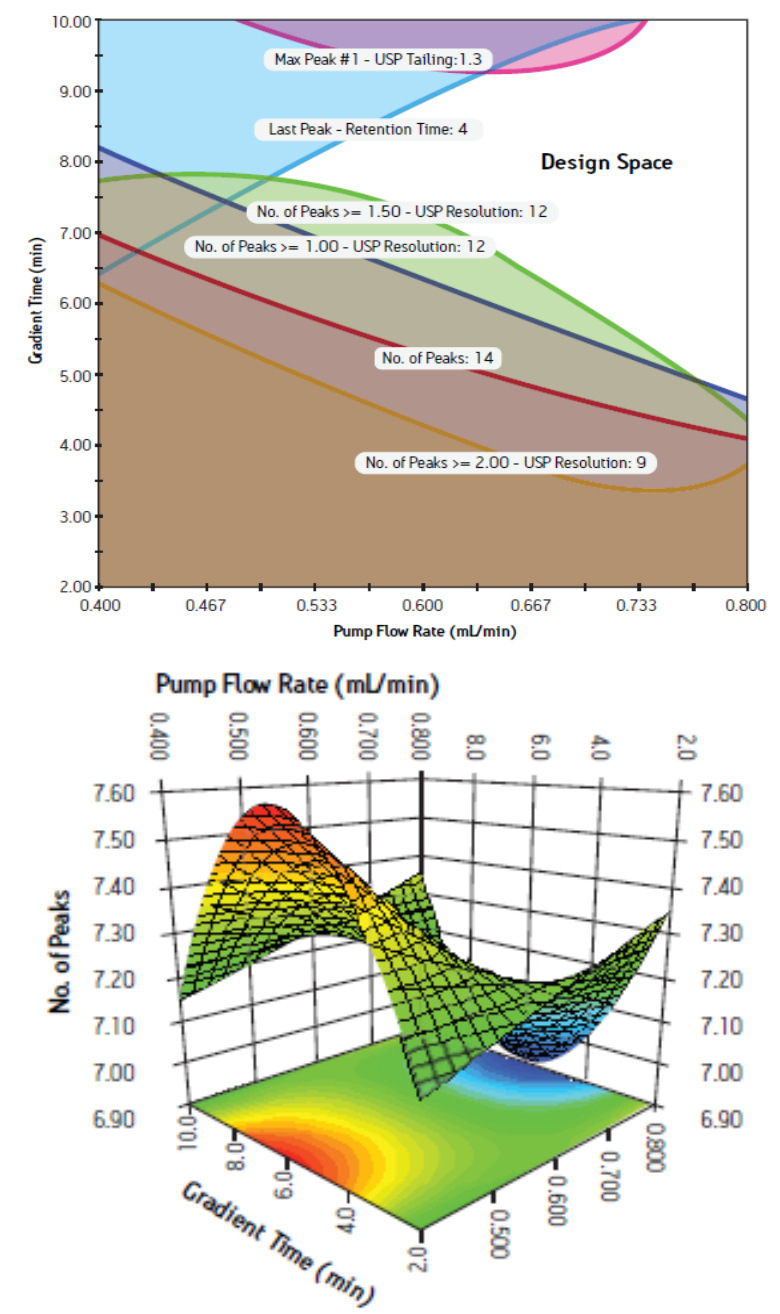

Fig. 3: The design space region showing the independent effects of gradient time and pump flow rate on method success. Data can also be visualized in 3D plots

\section{Mobile phase-B selection}

The compound was completely soluble in methanol. Upon the addition of small amount of mobile phase solution becomes hazy. Due to the haziness of the sample solution, it could precipitate inside the UHPLC column which was not suggestible. Moreover, various solvents, like acetonitrile, tetrahydrofuran and buffer combination has been used for selection of the organic modifier. This leads to a better selection as the miscibility and baseline issues were not observed in acetonitrile/methanol and buffer combination.

\section{Wavelength selection}

From the ultraviolet-visible spectra profiling, it was found that the suitable wavelength for dasatinib drug substance and its impurities were $258 \mathrm{~nm}$. Though the overlaid spectrum of the standard solution shows peak maxima's at $220 \mathrm{~nm}$ and $310 \mathrm{~nm}, 258 \mathrm{~nm}$ is selected based on the closeness of response of all impurities at this wavelength. At $220 \mathrm{~nm}$, it was observed that lower detection and unstable baseline. Whereas, different responses were observed at $310 \mathrm{~nm}$ which leads to a difference in the quantified and controlled manner of outcomes. Selection of $258 \mathrm{~nm}$ gives the almost similar; reproducible results of quantified and regularized style of results were observed, moreover uniform responses and baseline stability was good at $258 \mathrm{~nm}$. 


\section{Concluded method}

Several UHPLC methods aiming for shorter runtime and high throughput were for the separation of five impurities (including potential genotoxic impurities and other process intermediates) and dasatinib from each other. These includes different stationary phase, column dimension, and buffers. Considering the $\log \mathrm{P}$ values, $0.1 \%$ $\mathrm{v} / \mathrm{v}$ trifluoroacetic acid was selected as the buffering reagent for the quantification of potential genotoxic impurities of dasatinib. From various trails, $0.1 \% \mathrm{v} / \mathrm{v}$ trifluoroacetic acid as mobile phase-A and acetonitrile as mobile phase- $\mathrm{B}$ was selected and the gradient was optimized so that all the impurities and dasatinib peak were well separated from each other. No blank interference at the retention time of the peak of interest. The UHPLC method developed for the determination of quantitation of potential genotoxic impurities of dasatinib was precise, accurate and specific.

The method has been validated and found satisfactory results were observed for all the tested validation parameters. The chromatograms of standard and sample solutions were given in fig. 4 .
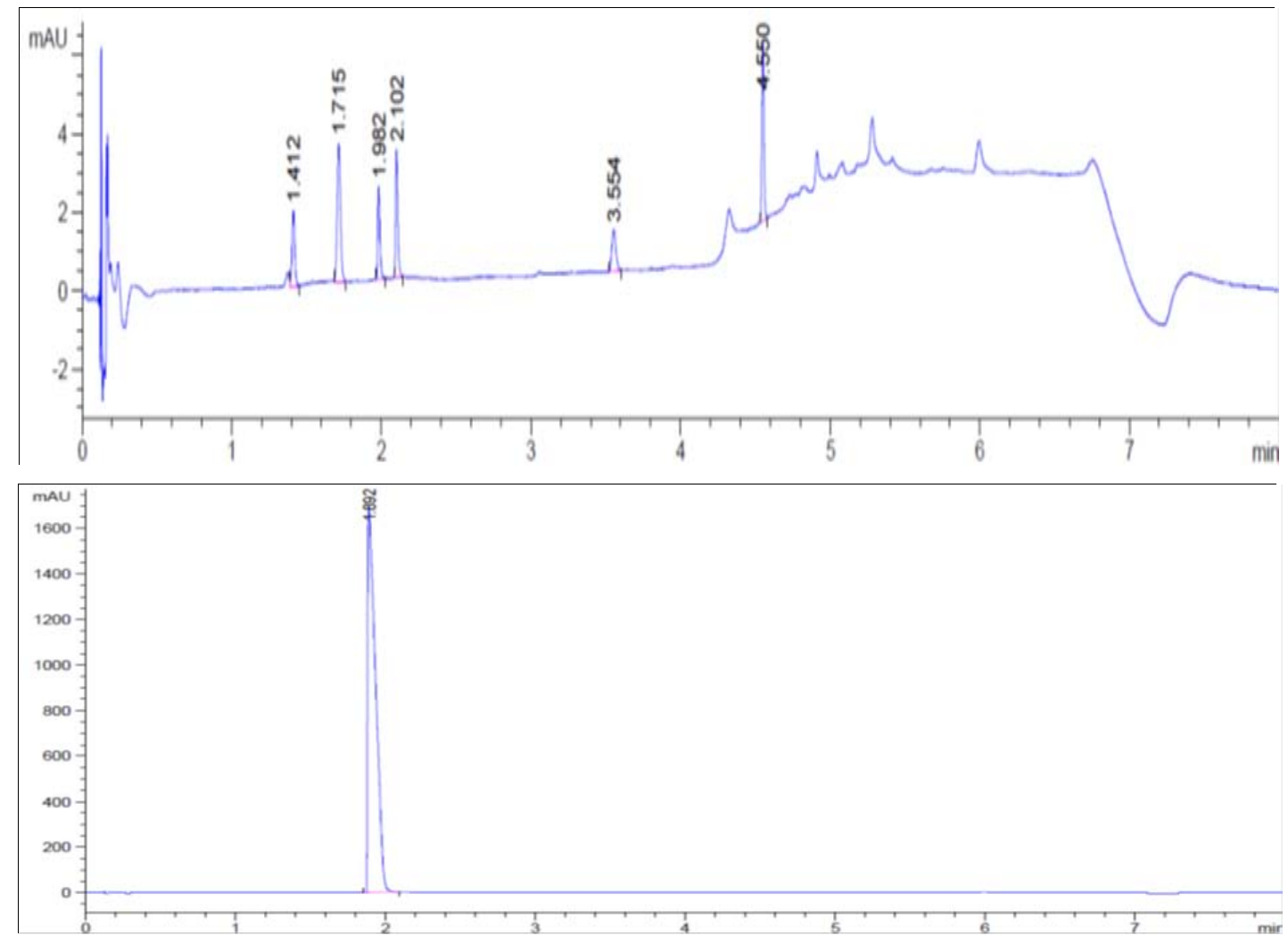

Fig. 4: Chromatograms of standard and sample solutions

\section{RESULTS AND DISCUSSION}

\section{Analytical method validation}

The method was validated for system suitability, system precision, method precision, detection limit, quantitation limit, linearity and range, recovery, intermediate precision, specificity/stress study, robustness and solution stability for standard, sample and mobile phase [18].

\section{System suitability}

System suitability of the method was assessed by the resolution between the peaks through injecting the standard solution which contains imp-1, imp-2, imp-3, dasatinib compound-A and compound-B. The resolution between the peaks was observed that more than resolution of 1.5 (acceptance criteria: resolution should be not less than 1.5). These results showed that the system was suitable and accepted for the determination of imp-1, imp-2 and imp-3 in dasatinib drug substance at the level of potentially genotoxic impurities. The resolution values have been given for all the peaks (from which it could also indicate the specificity of the method) in table 1 . The chromatogram of standard solution was shown in fig. 4.

\section{System precision}

The precision of an analytical procedure: expresses the closeness of agreement between a series of measurements obtained from multiple sampling of the same homogeneous sample under the prescribed conditions [18]. The same solution was injected six times and calculated the \% RSD for all peaks. \%RSD for all peaks was observed less than 5\% (acceptance criteria: \%RSD for peak area response should be not more than $15.0 \%$ ) shows that the system was precised for the determination of sample analysis and these results were accepted for imp-1, imp-2 and imp-3. The system precision results have been given in table 2 .

Table 1: Resolution between the peaks

\begin{tabular}{lll}
\hline Peak name & Retention time (min) & Resolution \\
\hline Imp-1 & 1.41 & - \\
Imp-2 & 1.71 & 8.4 \\
Dasatinib & 1.98 & 8.0 \\
Imp-3 & 2.10 & 4.7 \\
Compound-A & 3.55 & 39.4 \\
Compound-B & 4.54 & 27.2 \\
\hline
\end{tabular}


Table 2: System precision results of imp-1, imp-2, imp-3 and dasatinib

\begin{tabular}{|c|c|c|c|c|}
\hline \multirow[t]{2}{*}{ Injection no. } & \multicolumn{4}{|c|}{ Area response } \\
\hline & Imp-1 & Imp-2 & Imp-3 & Dasatinib \\
\hline 1 & 70260.3 & 60381.1 & 70134.4 & 55880.3 \\
\hline 2 & 64828.7 & 60336.7 & 65672.2 & 54828.7 \\
\hline 3 & 67799.0 & 61734.2 & 68181.1 & 57799.0 \\
\hline 4 & 67601.0 & 65631.6 & 68077.3 & 57601.0 \\
\hline 5 & 71472.1 & 60484.8 & 71417.7 & 52356.3 \\
\hline 6 & 69757.6 & 61198.8 & 65806.8 & 59757.6 \\
\hline Mean & 68619.8 & 61627.9 & 68214.9 & 56370.5 \\
\hline$\%$ RSD & 3.5 & 3.3 & 3.4 & 4.6 \\
\hline
\end{tabular}

\section{Method precision}

The precision of an analytical procedure: expresses the closeness of agreement between a series of measurements obtained from multiple sampling of the same homogeneous sample under the prescribed conditions [18]. The precision of the impurities was checked by injecting six individual preparations of impurities spiked at the concentration of $10 \mu \mathrm{g} / \mathrm{ml}$ level in the dasatinib drug substance sample. The \% RSD for percentage/content of imp-1, imp2 , imp-3 and dasatinib was below 3\% (acceptance criteria: \%RSD for percentage/content should be not more than 5.0\%). In the intermediate precision, the \%RSD for percentage/content impurities was within $2.5 \%$. These results indicated that the method was precise and accepted for the quantification of imp-1, imp-2 and imp3 in dasatinib drug substance. The precision method results were given in table 2 .

Table 3: Method precision results of imp-1, imp-2 and imp-3

\begin{tabular}{llll}
\hline Preparation no. & Imp-1 content $(\boldsymbol{\mu g} / \mathbf{m l})$ & Imp-2 content $(\boldsymbol{\mu g} / \mathbf{m l})$ & 10.2 \\
\hline 1 & 9.2 & 9.6 & 10.1 \\
2 & 9.2 & 9.8 & 10.1 \\
3 & 9.2 & 9.7 & 10.2 \\
4 & 9.2 & 9.6 & 9.6 \\
5 & 9.2 & 9.7 & 9.8 \\
6 & 9.1 & 9.8 & 10.0 \\
Mean & 9.2 & 9.7 & 2.4 \\
\hline
\end{tabular}

\section{Detection limit and quantitation limit}

The DL and QL for imp-1, imp-2, imp-3 and dasatinib determined based on signal-to-noise ratio method as per the ICH guideline Q2 (R1). For DL S/N ratio is 3:1 and QL S/N ratio is $10: 1$, by injecting a series of dilute solutions with known concentration DL/QL has been determined. The limit of detection for imp-1, imp-2, imp-3 and dasatinib were $0.08,0.09,0.07$ and $0.09 \mu \mathrm{g} / \mathrm{ml}$ for $2 \mu \mathrm{l}$ injection volume respectively. The limit of quantification for imp-1, imp-2, imp-3 and dasatinib were $0.24,0.27,0.21$ and $0.27 \mu \mathrm{g} / \mathrm{ml}$ for $2 \mu \mathrm{l}$ injection volume respectively. The precision study at the LOQ level performed. The \% RSD for the areas of each impurity was within $5.1 \%$. The DL and QL values show that the method was highly sensitive for the determination of imp-1, imp-2 and imp-3 in dasatinib drug substance.

\section{Linearity}

The linearity of analytical procedure: is its ability to obtain test results which are directly proportional to the concentration of an analyte in the sample [18]. The linearity of the method was evaluated by determining six concentration levels from LOQ to $150 \%$ of $1000 \mu \mathrm{g} / \mathrm{ml}$ analyte concentration. The correlation coefficient obtained for dasatinib was 0.9979. The best-fit linear equation obtained for dasatinib was $y=6007.4 x-520.38$. The correlation coefficient obtained for imp-1, imp-2 and imp-3 were greater than 0.993 . The best-fit linear equation obtained for imp- 1 was $y=7342.5 x-1562.4$, for imp- 2 was $y$ $=7532.8 \mathrm{x}-958.01$ and for imp-3 was $\mathrm{y}=5704.3 \mathrm{x}-760.56$. All the correlation coefficients were very close to one, so the developed and validated method was linear for the quantification of imp-1, imp-2 and imp-3 in dasatinib drug substance. The linearity graphs were shown in fig. 5-8. The data were given in table 4-7. The correlation coefficient square $\left(\mathrm{r}^{2}\right)$ for imp-1, imp-2, imp-3 and dasatinib were more than 0.990 . The linear regression data shows that the method was linear over the entire concentration, and it was adequate for its intended concentration range (QL to $150 \%$ ).

\section{Range}

The range was demonstrated in the interval $(0.3-14.7 \mu \mathrm{g} / \mathrm{ml})$ for imp-1, $(0.3-15.3 \mu \mathrm{g} / \mathrm{ml})$ for imp-2, $(0.3-15.2 \mu \mathrm{g} / \mathrm{ml})$ for imp-3 and $(0.3-15.4 \mu \mathrm{g} / \mathrm{ml})$ for dasatinib.

\section{Accuracy}

The accuracy of an analytical procedure: expresses the closeness of agreement between the value which is accepted either as a conventional true value or an accepted reference value and the value found. For the quantitative approaches, at least nine determinations across the specified range should be obtained [18]. Recovery study was carried out in triplicate at LOQ, 100 and $150 \%$ of the test concentration $(1000 \mu \mathrm{g} / \mathrm{ml})$. The percentage of recovery for imp-1, imp-2 and imp-3 were $90-95 \%$. Thus, the indicated method was suitable as the mean recovery value was well within the acceptance criteria $(80-120 \%)$ for the determination of quantification potential genotoxic impurities in drug substances.

Table 4: Linearity data of imp-1

\begin{tabular}{llll}
\hline Sample No. & \% Level & Concentration $(\boldsymbol{\mu g} / \mathbf{m l})$ & Peak response \\
\hline 1 & QL & 0.3 & 1651.9 \\
2 & 50 & 5.0 & 35246.5 \\
3 & 80 & 8.0 & 56041.6 \\
4 & 100 & 10.0 & 71556.9 \\
5 & 120 & 12.0 & 83229.9 \\
6 & 150 & 14.7 & 109685.6 \\
Slope & & 7342.5 & -1562.4 \\
Y-intercept & & 0.9966 & \\
Correlation co-efficient square $\left(\mathrm{r}^{2}\right)$ & & \\
\hline
\end{tabular}




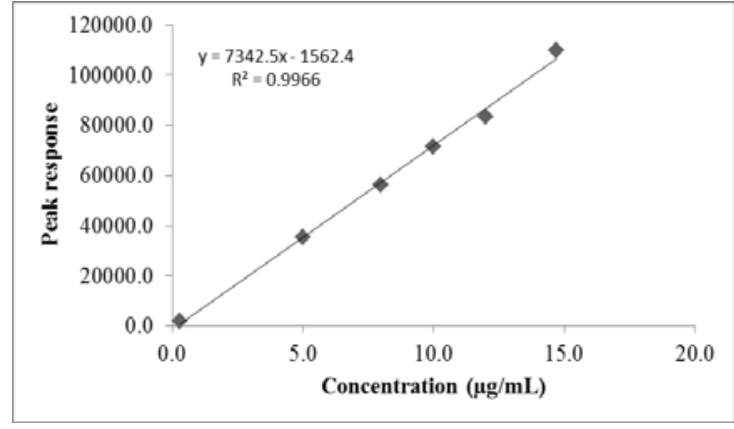

Fig. 5: Linearity graph for imp-1

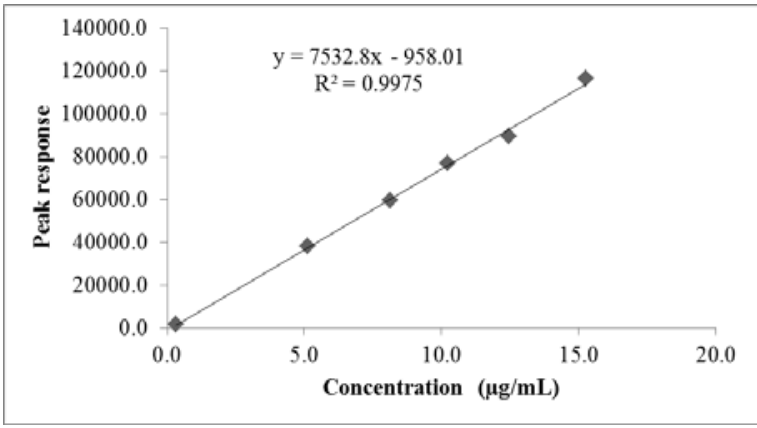

Fig. 6: Linearity graph for imp-2

Table 5: Linearity data of imp-2

\begin{tabular}{llll}
\hline Sample No. & \% Level & Concentration $(\boldsymbol{\mu g} / \mathbf{m l})$ & Peak response \\
\hline 1 & QL & 0.3 & 1677.8 \\
2 & 50 & 5.1 & 38184.9 \\
3 & 80 & 8.1 & 59600.3 \\
4 & 100 & 10.2 & 76703.5 \\
5 & 120 & 12.5 & 89364.1 \\
6 & 150 & 15.3 & 116542.0 \\
Slope & & 7532.8 & -958.01 \\
Y-intercept & & 0.9975 & \\
Correlation Co-efficient square $\left(\mathrm{r}^{2}\right)$ & & \\
\hline
\end{tabular}

Table 6: Linearity data of imp-3

\begin{tabular}{llll}
\hline Sample No. & \% Level & Concentration $(\boldsymbol{\mu g} / \mathbf{m l})$ & Peak response \\
\hline 1 & QL & 0.3 & 1324.9 \\
2 & 50 & 5.1 & 29601.7 \\
3 & 80 & 8.1 & 45700.6 \\
4 & 100 & 10.1 & 53336.6 \\
5 & 120 & 12.1 & 67249.8 \\
6 & 150 & 15.2 & 88668.2 \\
Slope & & 5704.3 & -760.56 \\
Y-intercept & & 0.9945 & \\
Correlation Co-efficient square $\left(\mathrm{r}^{2}\right)$ & & & \\
\hline
\end{tabular}

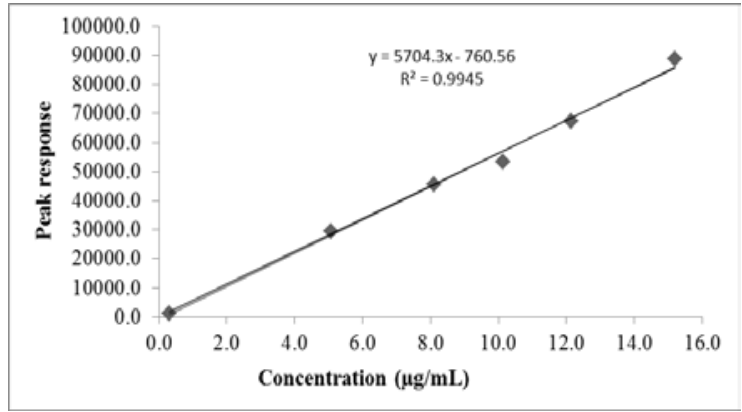

Fig. 7: Linearity graph for imp-3

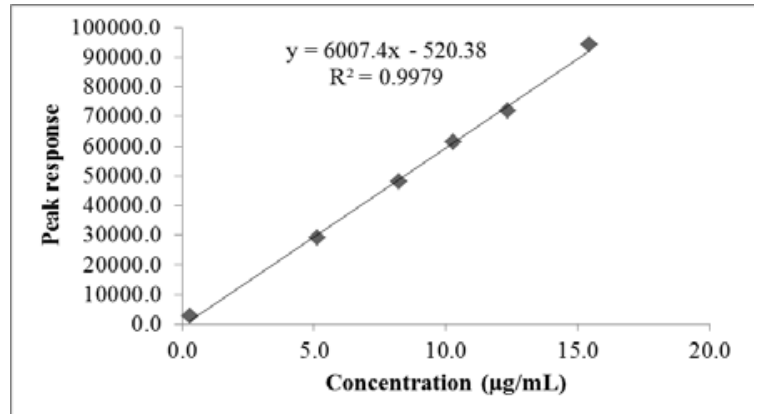

Fig. 8: Linearity graph for dasatinib

Table 7: Linearity data of dasatinib

\begin{tabular}{llll}
\hline Sample No. & \% Level & Concentration $(\boldsymbol{\mu g} / \mathbf{m l})$ & Peak response \\
\hline 1 & QL & 0.3 & 2890.5 \\
2 & 50 & 5.1 \\
3 & 80 & 8.2 \\
4 & 100 & 10.3 \\
5 & 120 & 12.4 & 48165.6 \\
6 & 150 & 15.4 & 61301.2 \\
Slope & & 6007.4 \\
Y-intercept & & -520.38 \\
Correlation Co-efficient square $\left(\mathrm{r}^{2}\right)$ & & 0.9979 \\
\hline
\end{tabular}




\section{Intermediate precision}

The intermediate precision of the method was evaluated by the different analyst, column and by using different equipment, \% RSD's were within $5 \%$, confirming the precision of the method.

These results indicated that the intermediate precision of this method was accepted for imp-1, imp-2, imp-3 and dasatinib.

\section{Specificity}

Specificity is the ability to assess the analyte unequivocally in the presence of components, which may be expected to be present. Typically, these might include impurities, degradants, matrix, etc. [18]. Diode array detection was used to show the specificity of the method which was assessed by performing forced degradation studies. The specificity of the developed UHPLC method for dasatinib was determined in the presence of potential genotoxic impurities namely imp-1, imp-2, imp-3. Also compound-A, B included to show the specificity of the method. Forced degradation studies were performed, and it shows the specificity and stability indicating a method for dasatinib. All stress decomposition studies were performed at a test concentration of $1000 \mu \mathrm{g} / \mathrm{ml}$. The peak purity analysis using a photodiode array detector demonstrates that the imp-1, imp-2 and imp-3 peaks were homogenous (i.e., the purity angle is lesser than the purity threshold). No interference was observed from diluent and impurities; at the retention time of imp-1, imp-2 and imp-3 peaks. During the specificity/forced degradation, it was observed that no secondary peak arising from degraded samples interfered with the elution of imp-1, imp-2 and imp-3 peaks. As a result, this method was well specific for the determination of imp-1, imp-2 and imp-3 in the presence of their degradation products.

\section{Results of stress studies}

Stress studies on dasatinib under different stress conditions (carried out as per ICH Q1B [21]), suggested the following degradation behavior. The drug was exposed to acid, base hydrolysis under reflux conditions. The dasatinib was not sensitive towards the treatment of acid and base hydrolysis. The drug was exposed to $3 \%$ hydrogen peroxide at room temperature for $24 \mathrm{~h}$. The dasatinib was not sensitive towards the treatment of hydrogen peroxide. No degradation observed. The drug was treated with water at $60^{\circ} \mathrm{C}$ for $24 \mathrm{~h}$. Not observed any degradation products were observed after $24 \mathrm{~h}$. The drug was stable towards water hydrolysis. The drug was sensitive to the effect of photolysis. When the drug powder was exposed to light for a completed exposure of $1.2 \times 10^{6}$ lux hours and an integrated near the ultraviolet energy of 200-Watt hours/square meter (in a photostability chamber), 13\% of degradation was observed. The drug was stable to the effect of temperature. When the compound was exposed to at $60^{\circ} \mathrm{C}$ for $10 \mathrm{~d}$ in a hot air oven, no degradation was observed. There was no interference of stressed samples with dasatinib and its impurities. Peak purity results for stressed dasatinib samples, derived from DAD detector, (the purity angle should be less than that of purity threshold) confirm that dasatinib, imp-1, imp-2 and imp-3 peaks were unique and pure. No degradation product peaks were observed after $8 \mathrm{~min}$ in the extended run time of $80 \mathrm{~min}$ for all the dasatinib stressed samples. Assay studies were carried out for stress samples against a qualified reference standard. The mass balance (addition of assay of drug substance and total impurities in drug substance) of stressed samples was close to $99.5 \%$ confirm the stability indicating the power of the developed method. From the degradation studies, it has been concluded that these potential genotoxic impurities were the process related impurities only not as degradation impurities.

\section{Robustness}

By cautious change in chromatographic conditions, the resolution between dasatinib, imp-1, imp-2 and imp-3 was evaluated. The mobile phase flow rate was $0.6 \mathrm{ml} / \mathrm{min}$. To check the effect of flow rate on the resolution, 0.1 units changed it from 0.5 to $0.7 \mathrm{ml} / \mathrm{min}$. The effect of column temperature on the resolution was studied at $35{ }^{\circ} \mathrm{C}$ and $25{ }^{\circ} \mathrm{C}$ instead of $30^{\circ} \mathrm{C}$. In all the careful varied chromatographic conditions carried out (flow rate, column temperature), the resolution between impurities and dasatinib was greater than 1.8, illustrating the robustness of the method. The system suitability results were meeting the acceptance criteria after individually changing the conditions of flow rate of mobile phase and column temperature (acceptance criteria are the resolution between the impurity peaks $>1.5$ and the ratio between the content of each impurity obtained from normal condition analysis and varied condition analysis should be within 0.70 to 1.30 ). The ratio of the content from normal and modified conditions met the acceptance criteria. Hence, the method was considered robust.

\section{Solution stability}

The solution stability of dasatinib and its potential genotoxic impurities was carried out by leaving spiked sample solution in a firmly closed volumetric flask at room temperature for $24 \mathrm{~h}$. The stability was carried out by freshly prepared sample solutions against freshly prepared reference standard solutions at $24 \mathrm{~h}$. From this solution stability; standard and sample solution were stable up to $24 \mathrm{~h}$.

\section{CONCLUSION}

A rapid, cost-effective infinity LC method was successfully developed for quantitative determination of potentially genotoxic impurities of dasatinib drug. The method was found to be precise and accurate with decent and constant recoveries. The authenticated method may be used for the regular analysis of the determination of potential genotoxic impurities of dasatinib drug substances in quality control laboratories.

\section{ABBREVIATION}

UHPLC: Ultra-High Performance Liquid Chromatograph, Imp: Impurity, $\mathrm{NaOH}$ : Sodium hydroxide, $\mathrm{HCl}$ : Hydrochloric acid, $\mathrm{H}_{2} \mathrm{O}_{2}$ : Hydrogen peroxide, ICH: International Conference on Harmonization, RSD: Relative Standard Deviation, DL/IOD: Limit of Detection, QL/IOQ: Limit of Quantification

\section{CONFLICT OF INTERESTS}

\section{Declared none}

\section{REFERENCES}

1. NIOSH Alert: Preventing occupational exposures to antineoplastic and other hazardous drugs in healthcare settings. US department of health and human Services, public health service, centers for disease control and prevention, national Institute for occupational; 2004. p. 1-29.

2. Safety and health, DHHS (NIOSH) publication; 2004. p. 165.

3. Mohammed G Kassem, Essam Ezzeldin, Hesham M Korashy, Gamal AE Mostafa. A high-performance liquid chromatographic method for the determination of dasatinib in rabbit plasma using fluorescence detection and its application to a pharmacokinetic study. J Chromatogr B 2013;939:73-9.

4. Michael TF, Shruti A, Dara H, Michael L, Steve U, Linda K, et al. A validated LC-MS/MS assay for the simultaneous determination of the anti-leukemic agent dasatinib and two pharmacologically active metabolites in human plasma: application to a clinical pharmacokinetic study. J Pharm Biomed Anal 2012;58:130-5.

5. Silvia DF, Antonio DA, Francesca DM, Elisa P, Lorena B, Marco S, et al. New HPLC-MS method for the simultaneous quantification of the antileukemia drugs imatinib, dasatinib, and nilotinib in human plasma. J Chromatogr B 2009;877:1721-6.

6. Rao KNV, Srivani P, Raja MA, Banji D, Kumar DS. Analytical method development and validation of dasatinib in its pharmaceutical dosage form by UPLC with forced degradation studies. Int J Pharm Res Scholars 2013;2:221-7.

7. Ramachandra B, Suguna B, Sivarami Reddy P, Naidu NVS. Development of a UV-visible spectrophotometric method for determination of dasatinib in pharmaceutical formulation and biological samples. Int J Pharm Sci Res 2015;6:293-303.

8. Ramachandra B, Naidu NVS. Validation of RP-HPLC method for estimation of dasatinib in bulk and its pharmaceutical dosage forms. Int J Pharm Biol Sci 2014;4:61-8.

9. Sreedevi A, Lakshmana Rao A. Development and validation of novel HPLC method for the estimation of dasatinib in bulk and 
pharmaceutical dosage forms. Int J Res Pharm Chem 2013;3:724-9.

10. Alagar Raja M, Namratha CH, David Banji, Rao KNV, Selvakumar D. RP-HPLC method for estimation of dasatinib in active pharmaceutical ingredient and pharmaceutical dosage form as per ICH guidelines. Asian J Pharm Anal Med Chem 2015;3:109-16.

11. Vivekananda Reddy D, Sreelatha P, Rama Devi B. A novel stability indicating assay method development and validation of dasatinib tablets formulations. Chem Sci Rev Lett 2014;3:747-58.

12. Antonio D'Avolio, Marco Simiele, Silvia De Francia, Alessandra Ariaudo, Lorena Baietto, Jessica Cusato, et al. HPLC-MS method for the simultaneous quantification of the antileukemia drugs imatinib, dasatinib and nilotinib in the human peripheral blood mononuclear cell (PBMC). J Pharm Biomed Anal 2012;59:109-16.

13. Thulase Nadh Reddy Dodda, Regalla Narasimha reddy, Meghana Reddy Regalla. Method development and validation of stability indicating RP-HPLC method for the estimation of dasatinib in tablet dosage form. Indo Am J Pharm Res 2013;3:1331-45.

14. Jiazhi Wang, Yunfang Zhou, Cheng Wang, Jianmiao Chen. Simultaneous determination of lapatinib, cabozantinib, imatinib, dasatinib, sorafenib, crizotinib, erlotinib and saha in rat plasma by UPLC-MS/MS. Latt Am J Pharm 2015;34:1576-84.

15. Yunfang Zhou, Shuanghu Wang, Ting Ding, Mingdong Wu, Peiwu Geng, Qingwei Zhang, et al. Pharmacokinetic interaction study of combining imatinib with dasatinib in rats by UPLCMS/MS. Drug Dev Ind Pharm 2015;41:1948-53.

16. Hari Babu B, Bala Murali Krishna K, Rama Krishnaveni P. Development and validation of HPLC method for the estimation of lapatinib in bulk drugs and pharmaceutical formulations. Int J Res Rev Pharm Appl Sci 2011;1:207-14.

17. Mhaske DV, Dhaneshwar SR. Stability indicating HPTLC and LC determination of dasatinib in pharmaceutical dosage form. Chromatographia 2007;66:1-2.
18. http://www.ich.org/fileadmin/Public_Web_Site/ICH_Products /Guidelines/Quality/Q2_R1/Step4/Q2_R1_Guideline.pdf. [Last accessed on 10 Jun 2016].

19. http://www.oecd.org/chemicalsafety/testing/oecdquantitatives tructure-activityrelationshipsprojectqsars.htm. [Last accessed on 10 Jun 2016].

20. Kunal Roy, Supratik Kar, Rudra Narayan Das. Understanding the basics of QSAR for applications in pharmaceutical sciences and risk assessment, Academic press, Elsevier, New York, 2015.

21. http://www.ich.org/fileadmin/Public_Web_Site/ICH_Products/G uidelines/Quality/Q1B/Step4/Q1B_Guideline.pdf. [Last accessed on 10 Jun 2016].

22. Uma G, Najila Banu A, Sathica Taj A, Josephine Benedit Bai U. Phytochemical screening and antibacterial activity of scoparia dulcis extracts. Asian J Pharm Clin Res 2014;7:130-3.

23. Anila KN, Emmanuel J. Clinical pharmacist, governed anticoagulation service in stroke unit of a tertiary care teaching hospital. Asian J Pharm Clin Res 2013;6:149-52.

24. Sivamani S, Benin Joseph, Bibhas Kar. Zebrafish: an emerging model system for drug discovery. Asian J Pharm Clin Res 2016;9:11-4.

25. http://www.ich.org/fileadmin/Public_Web_Site/ICH_Products/G uidelines/Multidisciplinary/M7/M7_Step_4.pdf. [Last accessed on 10 Jun 2016].

26. http://www.accessdata.fda.gov/drugsatfda_docs/nda/2006/0 21986s000_Sprycel_ClinPharmR.pdf. [Last accessed on 10 Jun 2016].

27. http://www.drugbank.ca/drugs/DB01254. [Last accessed on 10 Jun 2016].

\section{How to cite this article}

- N Balaji, Sayeeda Sultana. Trace level determination and quantification of potentially genotoxic impurities in dasatinib drug substance by UHPLC/infinity LC. Int J Pharm Pharm Sci 2016;8(10):209-216. 